\title{
Cryopreservation of a small number of human sperm using enzymatically fabricated, hollow hyaluronan microcapsules handled by conventional ICSI procedures
}

\author{
Kazuhisa Tomita ${ }^{1,3} \cdot$ Shinji Sakai $^{4} \cdot$ Mehdi Khanmohammadi $^{4} \cdot$ Takayuki Yamochi $^{1}$. \\ Shu Hashimoto ${ }^{1}$ Masayuki Anzai $^{3}$ - Yoshiharu Morimoto ${ }^{2}$. Masahito Taya ${ }^{4}$. \\ Yoshihiko Hosoi $^{3}$
}

Received: 14 September 2015 / Accepted: 6 January 2016/Published online: 19 January 2016

(C) Springer Science+Business Media New York 2016

\begin{abstract}
Purpose We investigated whether enzymatically fabricated hyaluronan (HA) microcapsules were feasible for use in the cryopreservation of a small number of sperm.

Methods HA microcapsules were fabricated using a system of water-immiscible fluid under laminar flow. Three sperm were injected into a hollow HA microcapsule using a micromanipulator. Capsules containing injected sperm were incubated in a freezing medium composed of sucrose as the cryoprotectant and then placed in a Cryotop ${ }^{\circledR}$ device and plunged into liquid nitrogen. After thawing, the capsule was degraded by hyaluronidase, and the recovery rate of sperm and their motility were investigated.

Results The HA microcapsule measuring $200 \mu \mathrm{m}$ in diameter and with a $30-\mu \mathrm{m}$ thick membrane was handled using a conventional intracytoplasmic sperm injection (ICSI) system, and
\end{abstract}

Capsule Hollow HA microcapsules can be used for the cryopreservation of a small number of sperm without producing adverse effects on sperm quality.

Kazuhisa Tomita

tomita@ivfnamba.com

1 IVF Namba Clinic, 1-17-28 Minamihorie, Nishi-ku, Osaka 550-0015, Japan

2 HORAC Grand Front Osaka Clinic, 3-1 Ofuka-cho, Kita-ku, Osaka 530-0011, Japan

3 Laboratory of Molecular Developmental Biology, Graduate School of Biology-Oriented Science and Technology, Kinki University, Wakayama 649-6493, Japan

4 Division of Chemical Engineering, Department of Materials Engineering Science, Graduate School of Engineering Science, Osaka University, 1-3 Machikaneyama-cho, Toyonaka, Osaka 560-8531, Japan the procedure involved the injection of sperm into the capsule. The HA microcapsules containing sperm were cryopreserved in a Cryotop ${ }^{\circledR}$ device and decomposed by the addition of hyaluronidase. The recovery rate of sperm after cryopreservation and degradation of HA microcapsules was sufficient for use in clinical practice (90\%).

Conclusions Hollow HA microcapsules can be used for the cryopreservation of a small number of sperm without producing adverse effects on sperm quality.

Keywords Small number of sperm $\cdot$ Human sperm cryopreservation $\cdot$ Microcapsule $\cdot$ Hyaluronan $\cdot$ ICSI

\section{Introduction}

The development of intracytoplasmic sperm injection (ICSI) has paved the way toward the fertilization of an oocyte using one sperm [1]. In addition, the combination of testicular sperm extraction (TESE) and ICSI has expanded the possibility of a successful pregnancy for patients with severe male factor infertility [2]. However, TESE does not always ensure successful sperm collection.

Cryopreservation of a small number of sperm is important to avoid unnecessary surgery for male and female patients because there is no guarantee of successful sperm collection following TESE treatment or in patients with severe oligozoospermia. Sperm cryopreservation is clinically performed before the cycle of oocyte pick up. Cryotubes or straws are generally used for sperm cryopreservation. In the procedure of cryopreservation and thawing using these vessels, the thawed sperm have to be diluted with a large volume of medium to eliminate cryoprotectants. As a result, the recovery rate of sperm might be extremely low. In addition, when ICSI is 
performed using a small number of sperm, it takes a much longer time until sperm injection, causing the accumulation of reactive oxygen species [3, 4]. Such changes in culture conditions have been shown to be involved in the lipid peroxidation of cell membranes, nuclear DNA fragmentation, and delay of embryo development [5-8]. Several groups have reported the cryopreservation of a small number of sperm using devices for embryo vitrification $[9,10]$ by embedding them into an alginate gel [11] and by injecting them into an empty zona pellucida (ZP) [12-14]. Sperm injection into an empty $\mathrm{ZP}$ is preferable to other methods because of low reproducibility in the vitrification device and reduced survival in the alginate gel. It is also easier for technicians compared with other methods because it is a similar procedure to ICSI [15]. However, there are several concerns over this technique because of the inadequate supply of a patients' own ZP, ethical problems of the use of ZP donation, and contamination of bacteria and viruses from animals.

To cope with these challenges, we aimed to fabricate a hollow spherical gel similar to a ZP to hold a small number of sperm. Here, we examined whether a hyaluronan (HA)-based microcapsule made through an enzymatic reaction can be manipulated for sperm injection and cryopreservation. Furthermore, the recovery rate and motility of sperm after cryopreservation in a microcapsule were investigated.

\section{Material and methods}

\section{Ethics}

This study was approved by the local ethics Institutional Review Board of IVF Namba Clinic. Sperm and unfertilized oocytes donated from patients who gave informed consent were used for the in vitro study.

\section{Preparation of HA microcapsules}

Sodium HA (MW: $1.79 \times 10^{6} \mathrm{Da}$, JNC Crop, Tokyo, Japan) was modified by phenolic moieties for the gelation of the HA solution. The HA derivative, possessing phenolic hydroxyl $(\mathrm{Ph})$ moieties (denoted as HA-Ph) at $1.6 \times 10^{-4} \mathrm{~mol}-\mathrm{Ph} / \mathrm{g}-\mathrm{HA}-\mathrm{Ph}$, was prepared based on a previously reported method through conjugation with tyramine via carbodiimide-mediated condensation in an aqueous solution [16].

Microparticles were fabricated using a co-flowing system under laminar flow [17]. In this system, a 26gauge tube (inner tube) was placed into a 21-gauge tube (outer tube). A paraffin stream was made by extrusion from the outer tube, and a stream of aqueous solution of microparticles flowed from the inner tube. The outer and inner tubes were connected to a compressor and syringe pump, respectively, and the flow rate of the two fluids could be adjusted. Droplet formation was obtained at the tip of the laminar flow, resulting in spherical gel fabrication by the co-flowing system.

For the production of the core of HA microcapsules, gelatin (Sigma, St. Louis, MO, USA) was dissolved at $7.5 \%(w / v)$ in phosphate-buffered saline (PBS) for extrusion from the inner tube. The gelatin solution flowed from the inner tube at a rate of $0.075 \mathrm{~mL} / \mathrm{min}$, and liquid paraffin containing $3 \%$ lecithin (Wako, Osaka, Japan) was extruded through the outer tube at a flow rate of $2.9 \mathrm{~mL} / \mathrm{min}$. The gelatin microparticles were collected in a $50-\mathrm{mL}$ plastic tube and cooled at $4{ }^{\circ} \mathrm{C}$ to complete gelation. The gelatin microparticles were rinsed with cooled PBS buffer for the removal of ambient liquid paraffin. Then, the HA-Ph solution $[0.75 \%(w / v)]$ was supplemented with horseradish peroxidase (HRP, Wako Pure Chemicals, Osaka, Japan) at 100 units $/ \mathrm{mL}$ and was mixed with the gelatin microparticles at a ratio of 10:1 (v/w) for the production of HA-Ph microcapsules with a gelatin core. The HA-Ph solution supplemented HRP, and gelatin microparticles were extruded from the inner tube at a rate of $0.075 \mathrm{~mL} / \mathrm{min}$ into a coflowing fluid of liquid paraffin containing $3 \%$ lecithin and $1.4 \mathrm{mM} \mathrm{H}_{2} \mathrm{O}_{2}$ (Wako Pure Chemicals, Osaka, Japan) at a flow rate of $1.8 \mathrm{~mL} / \mathrm{min}$. Through this reaction, gelatin microparticles were surrounded by gelated HA-Ph, and HA-Ph microcapsules with gelatin cores were obtained. HA-Ph microcapsules were collected in $50-\mathrm{mL}$ plastic tubes for $10 \mathrm{~min}$. The resultant emulsion was then left to stand for an additional $15 \mathrm{~min}$ to allow further cross-linking between the $\mathrm{Ph}$ moieties in $\mathrm{HA}-\mathrm{Ph}$. Finally, the microcapsules were rinsed with PBS buffer.

\section{Picking up of HA-Ph microcapsules from the prepared liquid}

Not all HA-based spherical vehicles contained gelatin microparticles. From the prepared samples, HA-Ph spherical vehicles with gelatin were picked up (Fig. 1a). One hundred microliters of the HA-Ph microcapsule sample was mixed with $1.0 \mathrm{~mL}$ of culture medium, and the microcapsules with gelatin cores were picked up using a glass pipet (Fig. 1b).

\section{Measurement of the diameter of HA-Ph microcapsules}

The diameter of HA-Ph microcapsules was measured using a ZILOS-tk ${ }^{\circledR}$ system (HAMILTON THORNE, Beverly, USA). Two different diameters of HA-Ph microcapsules and diameters of gelatin cores were measured. The average diameter of HA-Ph microcapsules and the length of the HA gel were calculated. The diameters of $38 \mathrm{HA}-\mathrm{Ph}$ microcapsules were measured, and the sizes of the capsules picked up were calculated. The average diameter of capsules was $241.6 \pm 30.4 \mu \mathrm{m}$ and 


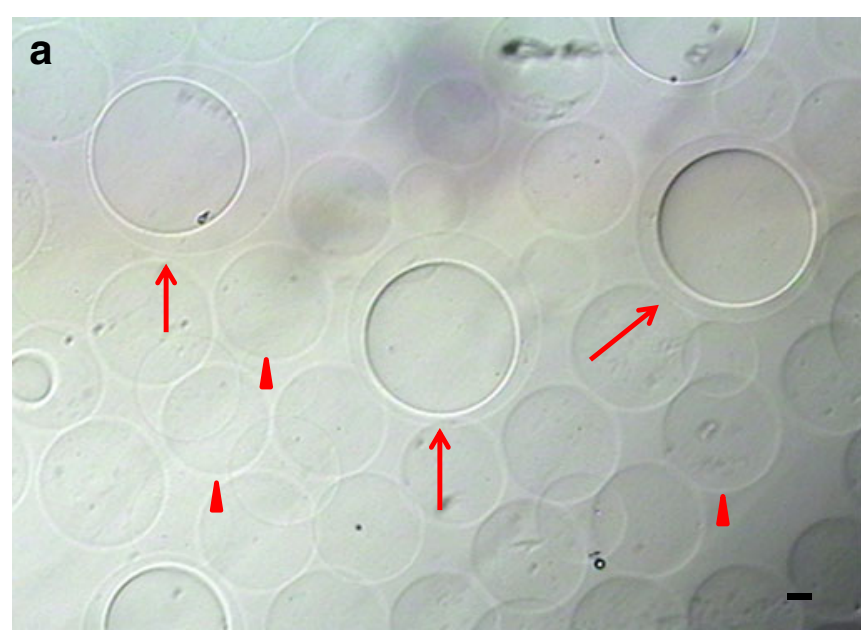

Fig. 1 Hyaluronan-phenolic hydroxyl (HA-Ph) microcapsules prepared using a water-immiscible co-flowing system. a The prepared sample using the co-flowing system. Arrow and arrow head indicate the HA

thickness of the HA membrane was $29.6 \pm 7.2 \mu \mathrm{m}$ $(N=38)$.

\section{Sperm preparation}

All sperm samples used in this study were surplus specimens from in vitro fertilization (IVF). The semen was collected by masturbation, liquefied for $30 \mathrm{~min}$ at $37^{\circ} \mathrm{C}$, and then centrifuged using 50-90\% Isorate ${ }^{\circledR}$ discontinuous density gradient for $20 \mathrm{~min}$ at $400 \mathrm{~g}$ (Irvine Scientific, Santana, USA). Washing using culture medium (GM501HTF, GYNEMED, Lensahn, Germany) was performed for $10 \mathrm{~min}$ at $400 \mathrm{~g}$. Subsequently, a swim-up method was performed by overlaying the culture medium $(0.5-1.0 \mathrm{~mL})$ above the obtained sample for 5 or $30 \mathrm{~min}$ at $37^{\circ} \mathrm{C}$. After fishing insemination, the surplus sample was used for experiment.

\section{Preparation of hollow HA-Ph microcapsules}

Aspiration and release of liquid by ICSI system in HA-Ph microcapsule with a gelatin could not be controlled freely. Thus, the gelatin core was degraded. To prepare hollow HA$\mathrm{Ph}$ microcapsules mimicking empty ZP (HA-ZP), the obtained capsules were treated with $0.4 \%$ trypsin-EDTA for $2 \mathrm{~min}$ at $37^{\circ} \mathrm{C}$ and washed in culture medium (sperm washing medium, Irvine, Santa Ana, USA) to stop the trypsin reaction. After degradation of the capsule cores by trypsin, no gelatin gel was observed in the capsules (Fig. 2). Then, to mark the HA-ZP, a drop of culture mineral oil was injected into each capsule.

\section{Sperm injection into the HA-ZP}

For the injection of sperm into the HA-ZP, a conventional holding pipet (Kitazato, Shizuoka, Japan) and

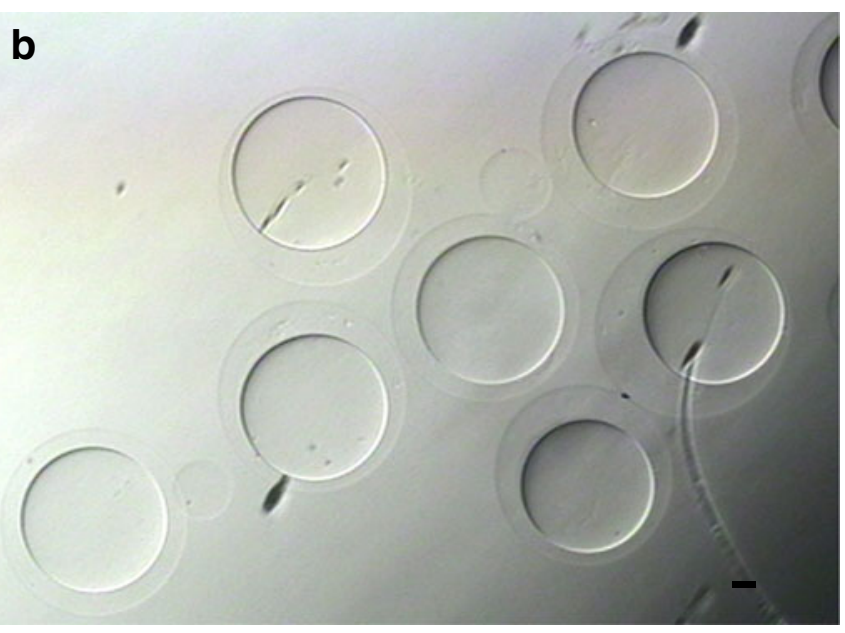

gel with or without gelatin core, respectively. b The HA-Ph microcapsule with gelatin core collected from a prepared sample. Scale bars $=20 \mu \mathrm{m}$

injection pipet (Sunlight Medical, Florida, USA) were used. The micromanipulator was a Narishige system (Narishige, Tokyo, Japan). Injection was performed using a conventional ICSI procedure. To confirm the morphology, sperm were mixed with $10 \%$ polyvinylpyroridone (PVP; Kitazato, Shizuoka, Japan). Only sperm with a normal morphology and progressive motility were injected. The HA-ZP was not penetrated until the needle touched the opposite side of the HA-ZP (Fig. 3a-c). After the HA-ZP penetration, the gel was restored to its original state, and a spherical shape was recovered (Fig. 3d). The HA-ZP with injected sperm was then degraded using $40 \mathrm{IU} / \mathrm{mL}$ hyaluronidase for about $10 \mathrm{~min}$ at $37{ }^{\circ} \mathrm{C}$ (Fig. 4).

\section{Analysis of motility and viability after treatment with hyaluronidase}

The effect of hyaluronidase treatment on sperm motility and viability was investigated. Four surplus specimens after IVF were used in this investigation. Conventional original semen data was below: total concentration of $42.1 \pm 7.1 \times 10^{6}$ cells/ $\mathrm{mL}$, motile rate of $54.5 \pm 5.2 \%$, and an abnormal morphology rate of $30.2 \pm 3.2 \%$, respectively. A total of $4.0 \times 10^{6} \mathrm{sperm} /$ $\mathrm{mL}$ was incubated in $40 \mathrm{IU} / \mathrm{mL}$ of hyaluronidase (Irvine Scientific, Santa Ana, USA) in culture medium for $1 \mathrm{~h}$ at $37^{\circ} \mathrm{C}$. As a control, sperm were incubated in the same culture media (sperm washing medium, Irvine) and conditions. Sperm motility and viability between the hyaluronidase treated and non-treated groups were compared. Sperm motility was assessed using a Macklar chamber ${ }^{\circledR}$ (Sefimedical Instruments Ltd., Haifa, Israel) under $\times 200$ magnification. Sperm with progressive and nonprogressive motility were counted and used to obtain the percentage of motile sperm. Viability was assessed 


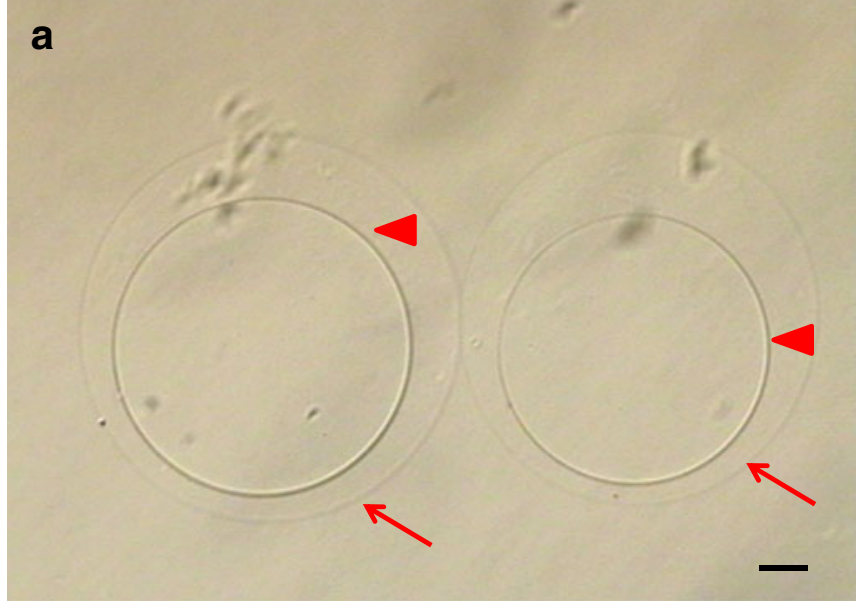

Fig. 2 Treatment of hyaluronan-phenolic hydroxyl (HA-Ph) microcapusles with gelatin cores by trypsin. a Before and $\mathbf{b}$ after treatment by trypsin. Arrow head and arrow in a indicate the contour of the gelatin core and HA gel, respectively. After treatment with trypsin, the

using the hypo-osmotic swelling test in $150 \mathrm{mOsm} / \mathrm{kg}$ solution [18]. The morphology of 100 sperm were
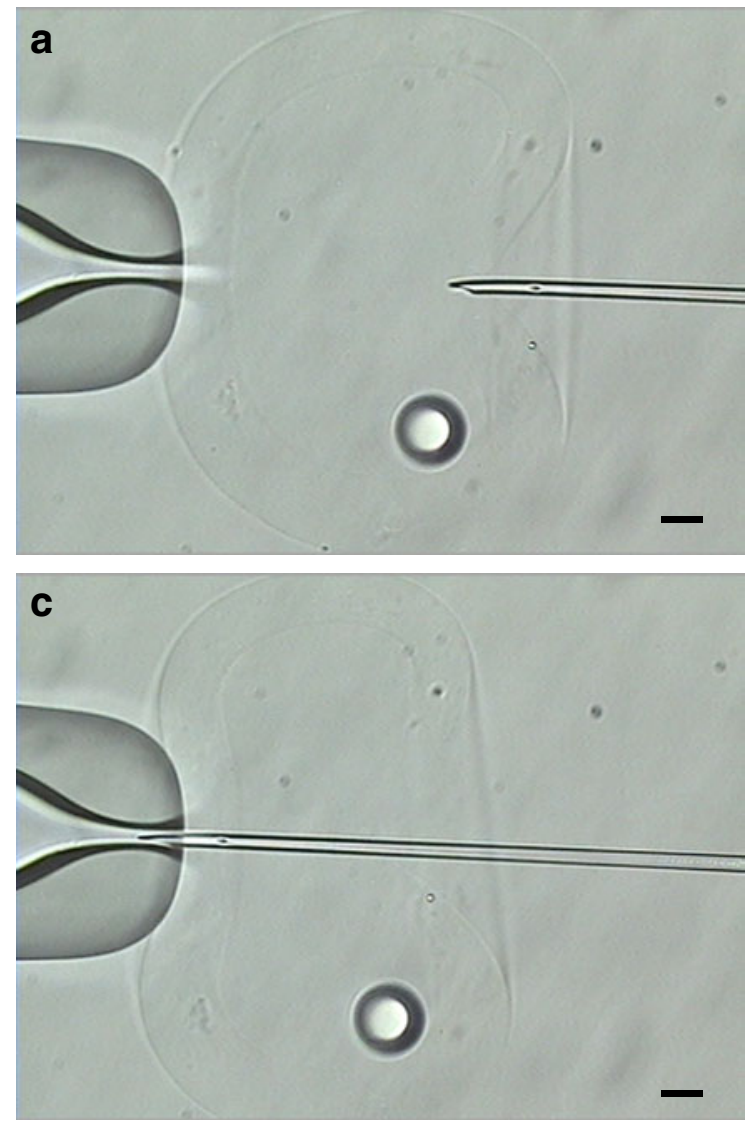

Fig. 3 Injection of sperm to hollow hyaluronan-microcapsule mimicking zona pellucida (HA-ZP) by a conventional intracytoplasmic sperm injection (ICSI) procedure (a-b) HA gel was pushed by the injection needle. The capsule morphology was changed by the injection force, but the HA gel has not penetrated at position (b). c HA gel has

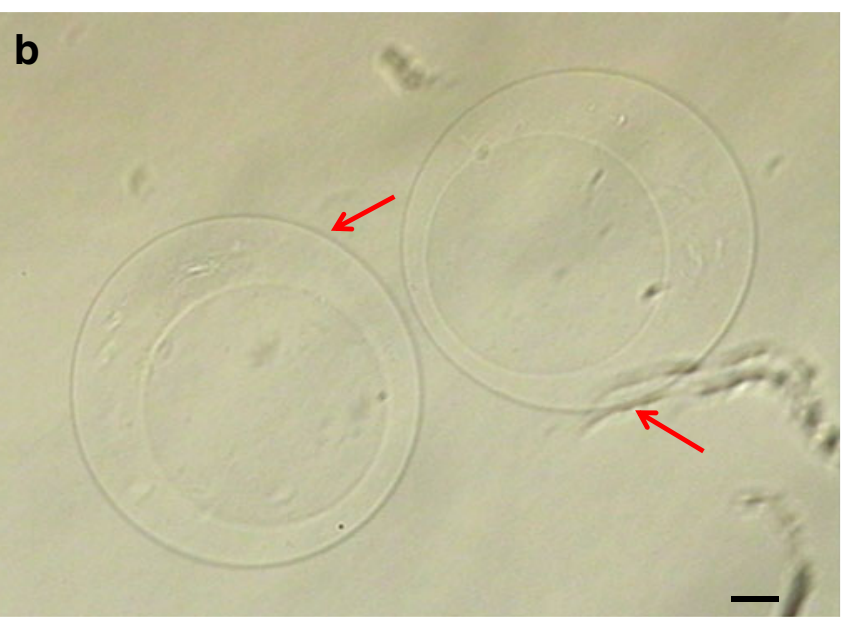

contour of the HA gel was observed (arrow in b), but the contour of the gelatin core (arrow head in a) was not observed. The decomposition of gelatin gel was confirmed morphologically. Scale bars $=20 \mu \mathrm{m}$

observed under $\times 400$ magnification. Sperm with swollen tails were decided as viable.
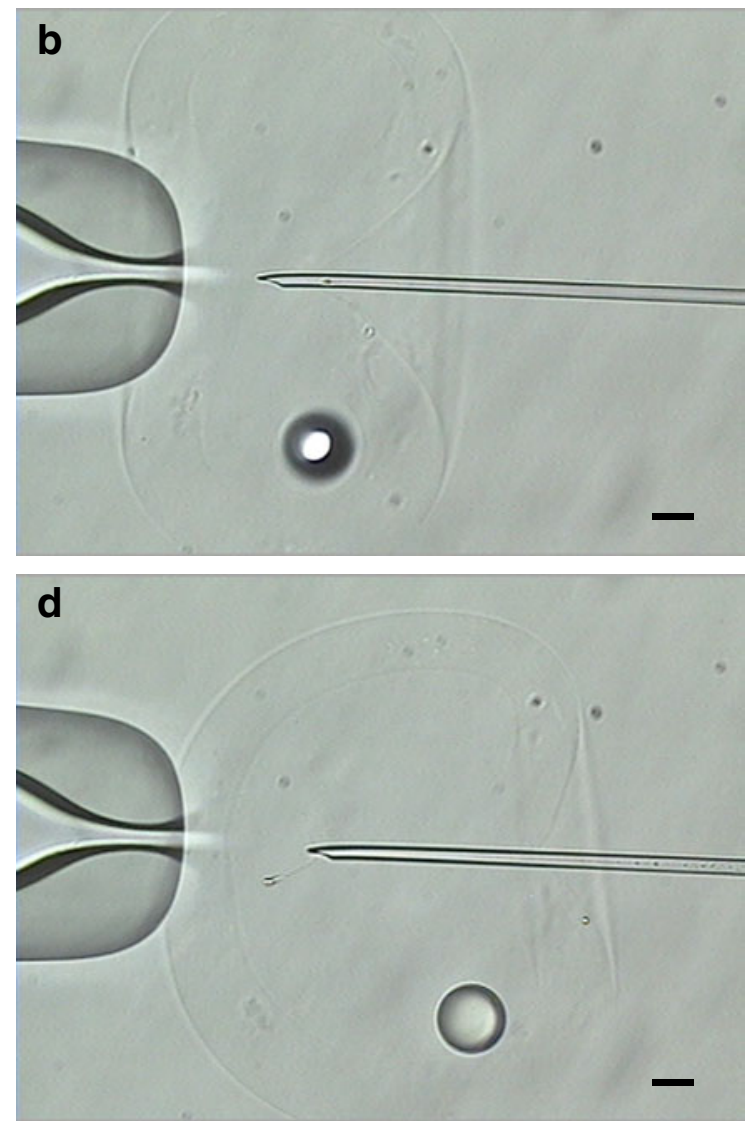

penetrated in the position where the injection needle touches the opposite side of the HA gel. d The penetrated HA-ZP returns to a spherical shape. The small sphere in the capsule indicates a drop of mineral oil injected before sperm injection. Scale bars $=20 \mu \mathrm{m}$ 

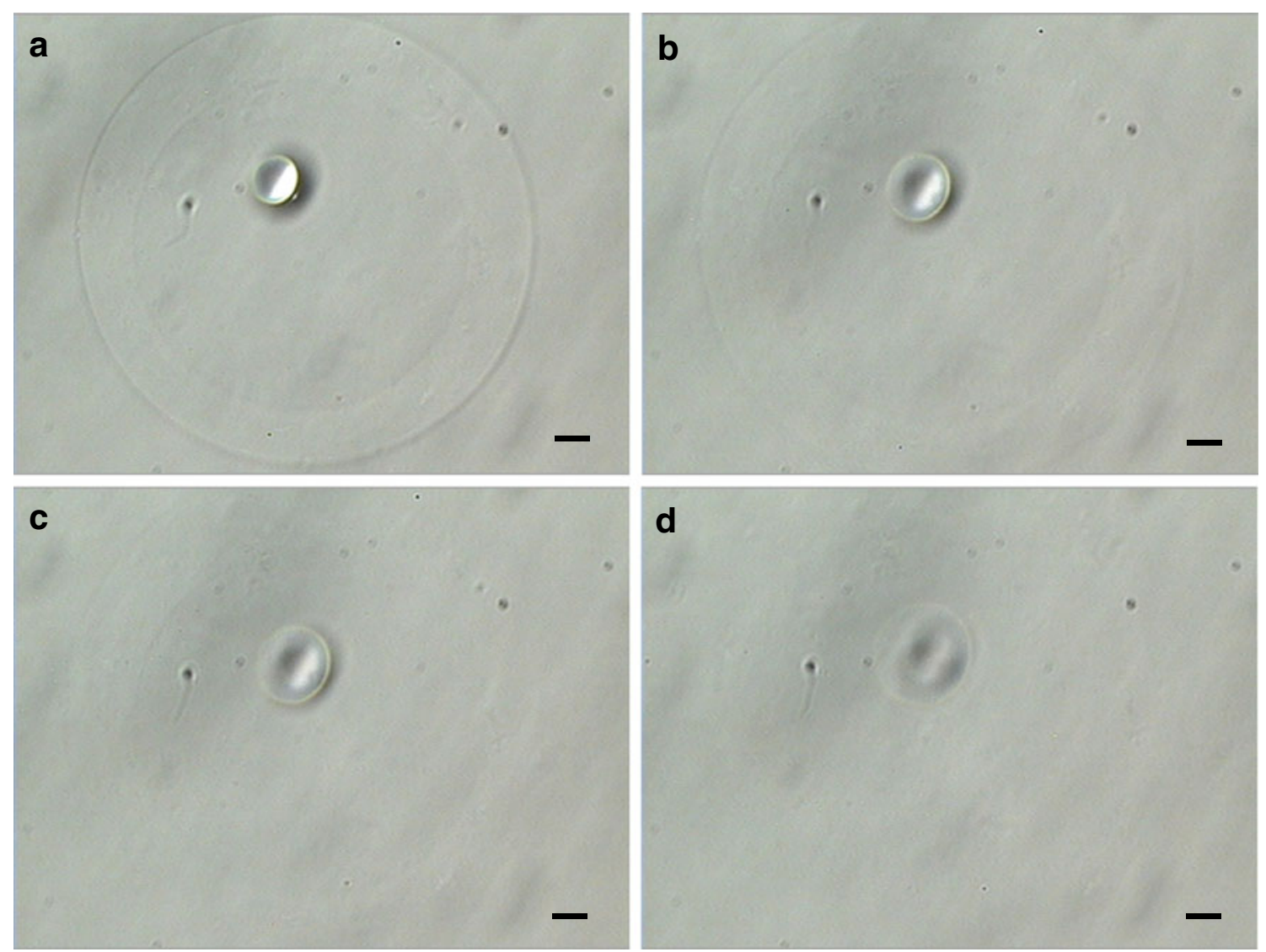

Fig. 4 Degradation of hollow hyaluronan microcapsule mimicking zona pellucida (HA-ZP) by hyaluronidase. The pictures showed the HA-ZP treated by hyaluronidase for a $0, \mathbf{b} 5, \mathbf{c} 7$, and $\mathbf{d} 10 \mathrm{~min}$. HA microcapsules were degraded for approximately $10 \mathrm{~min}$. Scale bars $=20 \mu \mathrm{m}$

\section{Analysis of sperm motility rate after injection into the HA-ZP without cryopreservation}

Four surplus specimens were used in this analysis. Conventional semen data was below: total concentration of $53.2 \pm 25.8 \times 10^{6}$ cells $/ \mathrm{mL}$, motile rate of $57.6 \pm 19.5 \%$, and an abnormal morphology rate of $33.1 \pm 7.0 \%$. The sperm motility and recovery rates after injection into the HA-ZP without freezing was investigated. Three sperm were injected into an HA-ZP, and two microcapsules per patient were analyzed. The HA-ZP with sperm was incubated for $30 \mathrm{~min}$ at $37^{\circ} \mathrm{C}$. Then, the motility and recovery rates for sperm before and after incubation and after HA-ZP decomposition were compared. Empty ZP were prepared and used as controls. In the case of empty ZP, the motility and recovery were assessed after sperm aspiration from the ZP. Sperm with progressive and non-progressive motility were counted and used to obtain the percentage of motile sperm.

\section{Analysis of sperm motility and recovery rates after freeze-thawing and degradation of the HA-ZP}

Surplus sperm samples from 22 patients after IVF were subjected to this study. Conventional original semen data was below: total concentration of $60.5 \pm 33.8 \times 10^{6} \mathrm{cells} / \mathrm{mL}$, motile rate of $59.4 \pm 17.2 \%$, and an abnormal morphology rate of $35.3 \pm 10.8 \%$, respectively. To evaluate the sperm motility and recovery rates after thawing, three sperm were injected into an HA-ZP and empty ZP as the control, respectively. Sperm with progressive motility and normal morphology were injected.

Freezing of an HA-ZP or empty ZP was done base on rapid freezing method of sperm by using sugar as cryoprotectant. To substitute culture medium into the cryopreservation medium, the HA-ZP or empty ZP-injected sperm were incubated in $10 \mu \mathrm{L}$ droplets of cryopreservation medium for $5 \mathrm{~min}$ at room temperature. The composition of cryopreservation medium was decided based on previous report on sperm freezing by sucrose [19-23]. The cryoprotectant was $0.1 \mathrm{M}$ sucrose (Wako Pure Chemicals, Osaka, Japan) and $20 \%$ synthetic serum substitute (SSS, Irvine Scientific, Santana, USA) in culture medium (sperm washing medium, Irvine). The treated vessels were placed on the Cryotop ${ }^{\circledR}$ devices with approximately $1.0 \mu \mathrm{L}$ of cryopreservation medium using a glass pipet, and were put at $4.5 \mathrm{~cm}$ above the surface of liquid nitrogen $\left(\mathrm{LN}_{2}\right)$ for 2 min. The HA-ZP or empty ZP with sperm were then plunged into $\mathrm{LN}_{2}$ and cryopreserved for at least $24 \mathrm{~h}$. 
Thawing was performed at $37{ }^{\circ} \mathrm{C}$ in culture medium (sperm washing medium, Irvine) for $5 \mathrm{~min}$.

After freezing-thawing of the HA-ZP, the capsules were decomposed by incubation in $10 \mu \mathrm{L}$ drop of $40 \mathrm{IU} / \mathrm{mL}$ hyaluronidase covered by culture oil for $10 \mathrm{~min}$ at $37^{\circ} \mathrm{C}$. The recovered and motile sperm were counted after decomposition of HA-ZP. Sperm with progressive or non-progressive motility were decided as motile sperm, and total number of both motility states was used to obtain the percentage of motile sperm.

In the control group (empty ZP), recovered and motile sperm were counted after aspiration from the ZP using an injection pipet. The sperm with motility after aspiration is decided as motile (total number of progress and nonprogress motility). The rate of motile sperm was calculated as given below:

The rate of motile sperm $(\%)=($ number of sperm with motility/number recovered sperm) $\times 100$.

\section{Sperm motility after freezing in different size of HA-ZP}

Eleven specimens after IVF were used in this study. Conventional original semen data was below: total concentration $47.3 \pm 33.8 \times 10^{6}$ cells $/ \mathrm{mL}$; motile rate $53.3 \pm 18.4 \%$; abnormal morphology rate; $36.4 \pm 11.7 \%$, respectively. There was size difference in several tens of micrometers in fabricated HA-ZP. In this investigation, the smaller and larger HA capsules were divided from prepared sample, and motility after freezing was compared between two groups. The diameter and thickness of HA gel in small capsules were 270.0 $\pm 12.4 \mu \mathrm{m}$ and $29.3 \pm 8.1 \mu \mathrm{m}$, and large capsules were 327.0 $\pm 13.1 \mu \mathrm{m}$ and $22.8 \pm 8.6 \mu \mathrm{m}$, respectively. The freezingthawing procedure was performed, same as described above. Regarding of evaluation of sperm motility, the sperm with progressive or non-progressive motility were severally assessed. The rate of each state of motility was calculated as follows:

the rate of progressive or non-progressive motile sperm $(\%)=($ number of sperm with progressive or non-progressive motility/number of total motile sperm) $\times 100$.

In addition, to investigate whether the immotile sperm can be injected to oocyte, obtained immotile sperm after thawing was incubated in $10 \mu \mathrm{L}$ of $3.6 \mathrm{mM}$ pentoxifylline (Sigma Aldrich, Saint Louis, USA) in culture medium for $30 \mathrm{~min}$ at $37^{\circ} \mathrm{C}$, and motility recovery was assessed [24].

\section{Preparation of empty ZP}

As a control for the experiment, which assessed the capacity of cryopreservation of HA microcapsules, empty ZP extracted cytoplasm from oocyte was utilized. These oocytes were derived from unsuccessful ICSI attempts. The oocytes were treated with $17 \mu \mathrm{g} / \mathrm{mL}$ cytochalasin B (Sigma Aldrich, Saint
Louis, USA). The cytoplasm was extracted using a micromanipulator. The pipet for cytoplasm extraction was a normal injection needle for fertilization. After cytoplasm extraction, the ZP was washed three times in a $10 \mu \mathrm{L}$ drop of culture medium.

\section{Time for a sperm searching in an HA-ZP}

The specimens derived from three different patients were used in this study. Conventional original semen data was below: total concentration $60.8 \pm 26.1 \times 10^{6}$ cells $/ \mathrm{mL}$; motile rate $63.0 \pm 9.5 \%$; abnormal morphology rate; $30.7 \pm 6.1 \%$, respectively. Three motile sperm were injected into an HA-ZP, which was then incubated in $3 \mu \mathrm{L}$ of $40 \mathrm{IU} / \mathrm{mL}$ hyaluronidase drops covered by mineral oil. After capsule decomposition, each sperm was isolated under $\times 400$ magnification in a drop of hyaluronidase solution. The time taken from the initiation of sperm searching to immobilization in the PVP drop was recorded. The data was measured for three sperm for each patient. As a control, sperm specimens diluted at concentrations of 0.1 (general sperm concentration for ICSI) and $0.0001 \times 10^{6}$ cells $/ \mathrm{mL}$ (low concentration) were used. Three microliters of sperm sample were covered by mineral oil, and searching time was similarly recorded. Searching was performed for a maximum of three times in one drop of sperm sample. If sperm could not be found, an additional sperm sample was placed on the dish until three sperm were found.

\section{Statistical analysis}

The differences between two groups and among three groups were analyzed by $t$ test and the Tukey-Kramer test following analysis of variance (ANOVA), respectively. Statistical analysis was performed by Statview version 5 (SAS Institute Inc, Cary, NC, USA) and $P<0.05$ was considered to be significant. The data are given as mean values $\pm \mathrm{SD}$.

\section{Results}

\section{Influence of hyaluronidase treatment on sperm motility and viability}

To investigate whether hyaluronidase could be used for microcapsule decomposition, the effect of hyaluronidase treatment on the motility and viability of sperm was assessed without cryopreservation. No significant differences in motility and viability were observed between the hyaluronidase treated and non-treated groups (motility: $95.1 \pm 4.9$ vs. $84.7 \pm 9.2 \%$; viability: $92.8 \pm 7.7$ vs. $93.8 \pm 8.7 \%$, respectively, $N=4$ ). 


\section{Influence of sperm injection into the HA-ZP on sperm motility}

We assessed whether sperm injection into the HA-ZP affected sperm motility. All sperm injected into the HA-ZP could be held in the microcapsules after incubation. After decomposition of the HA-ZP, almost all sperm $(95.9 \pm 8.3 \%)$ were recovered (vs. empty ZP: $95.9 \pm 8.3 \%$ ). Sperm injected into an HA-ZP retained their motility after incubation $(95.9 \pm 8.3 \%)$ and decomposition of the microcapsule $(95.9 \pm 8.3 \%)$. These values were not different, compared with empty $\mathrm{ZP}$ groups (after incubation: 95.9 $\pm 8.3 \%$; decomposition: $95.9 \pm 8.3 \%, N=4)$.

\section{Motility and recovery rate of sperm after freeze-thawing and decomposition of the HA-ZP}

To analyze the efficacy of sperm cryopreservation with the HA-ZP, the motility and recovery rates of sperm after cryopreservation were compared with empty ZP controls. All frozen microcapsules were recovered after thawing (Table. 1). There was no difference in the recovery rate of sperm between the HA-ZP and empty ZP groups (95.5 vs. $93.9 \%$ ). The sperm motility rates were also not different between the two groups (13.6 vs. $15.1 \%)$.

\section{Sperm motility after freezing-thawing in different size of HA-ZP}

To investigate whether the size of HA-ZP affects to motility of sperm after freezing, sperm were injected to small $(270 \mu \mathrm{m})$ and large size $(330 \mu \mathrm{m})$ of HA-ZP, and motility after thawing was compared (Table. 2). Total motility after freezing-thawing of capsules was not different between small and large HA-ZP (12.1 vs. $15.1 \%)$. The grade of motility in motile sperm was not different between two groups (progressive motility: 50.0 vs. $20.0 \%$, non-progressive motility: 50.0 vs. $80.0 \%$, respectively). In the regard of motility recovery in immotile sperm after treated by pentoxifylline, there was no difference between small and large HA-ZP (3 vs. $0 \%$ ).

\section{Searching time for a sperm held in an HA-ZP}

We compared the searching time for sperm using the HA-ZP method with that of a sperm suspension for ICSI (general concentration: $0.1 \times 10^{6}$ and low concentration: $0.0001 \times 10^{6}$ cells $/ \mathrm{mL}$, respectively). The searching time for sperm with the HA-ZP $(2.5 \mathrm{~min})$ was significantly shorter than that for the low sperm concentration (26.6 min) (Fig. 5).

\section{Discussion}

In this study, we developed HA microcapsules that were similar to ZP. HA microcapsule fabricated by co-flowing system under laminar flow was composed of spherical HA gel with core of gelatin gel. Hollow HA microcapsules were prepared by decomposing the gelatin core of the capsule. A small number of sperm were successfully injected into the hollow HA microcapsule using conventional ICSI procedures. After freezing and thawing of the microcapsule containing sperm, most of the sperm were recovered after decomposition of the HA gel by hyaluronidase.

The HA microcapsules were prepared using waterimmiscible fluid under laminar flow. This technique has been already utilized in the research of microcapsules [25] and has many advantages as follows: (1) easy size control by controlling of the flow rate of liquid in the order of micrometers [26], (2) small variations in their size compared with droplet preparation methods using a magnetic stirrer and a homogenizer $[27,28]$, and (3) easy gelation due to modification by the phenol moiety and gelation by the peroxidase-catalyzed reaction in the laminar flow [29] because elemental HA cannot be gelated. This adjustable size range and molecular polymerization in the co-flowing system enabled the development of HA microcapsules of similar size to embryos. The capsules prepared by this system were the same size as expanded blastocysts; therefore, they were easily manipulated under an inverted microscope.

We considered sperm recovery by degradation of the capsule as a good method because it did not inhibit the general

Table 1 Recovery rate of capsule and sperm and motility after freeze-thawing in hollow hyaluronan microcapsule mimicking zona pellucida (HA-ZP)

\begin{tabular}{llll}
\hline & Recovery rate of capsule (\%) (number) & Recovery rate of sperm (\%) (number) & Motility after thawing and decomposition (\%) (number) \\
\hline HA-ZP & $100.0(22 / 22)$ & $95.5 \pm 11.7(63 / 66)$ & $13.6 \pm 16.8(9 / 63)$ \\
ZP & $100.0(22 / 22)$ & $93.9 \pm 13.1(61 / 66)$ & $15.1 \pm 19.9(10 / 62)$ \\
\hline
\end{tabular}

Specimens derived from 22 patients were used in this experiment. Conventional semen parameters subjected to this analysis: total concentration of 60.5 $\pm 33.8 \times 10^{6}$ cells $/ \mathrm{mL}$, motile rate of $59.4 \pm 17.2 \%$, an an abnormal morphology rate of $35.3 \pm 10.8$

Sperm with progressive and non-progressive motility was decided as the motile sperm

There was no significant difference in the recovery rate of sperm and motility in HA capsules compared with control

$H A-Z P$ hollow hyaluronan-phenolic hydroxyl (HA-Ph) microcapsule mimicking ZP, ZP empty zona pellucida $(N=22$, mean $\pm \mathrm{SD})$ 
Table 2 Motility after freezing-thawing in different size of hollow hyaluronan microcapsule mimicking zona pellucida (HA-ZP)

\begin{tabular}{|c|c|c|c|c|}
\hline (\%) (number) & $\mathrm{ZP}(\mathrm{D}: 164.8 \pm 7.9 \mu \mathrm{m})$ & $\begin{array}{l}\text { small HA-ZP (D: } 270.0 \pm 12.4 \mu \mathrm{m}) \\
(\mathrm{M}: 29.3 \pm 8.1 \mu \mathrm{m})\end{array}$ & $\begin{array}{l}\text { large HA-ZP }(\mathrm{D}: 327.0 \pm 13.1 \mu \mathrm{m}) \\
(\mathrm{M}: 22.8 . \pm 8.6 \mu \mathrm{m})\end{array}$ & $\mathrm{P}$ \\
\hline Device recovery & $100(11 / 11)$ & $100(11 / 11)$ & $100(11 / 11)$ & n.s \\
\hline Sperm recovery & $94.0 \pm 13.4(29 / 33)$ & $100(33 / 33)$ & $100(33 / 33)$ & n.s \\
\hline Total motility & $9.1 \pm 21.6(3 / 29)$ & $12.1 \pm 16.8(4 / 33)$ & $15.1 \pm 17.4(5 / 33)$ & n.s \\
\hline Progressive motility & $33.3(1 / 3)$ & $50.0(2 / 4)$ & $20.0(1 / 5)$ & n.s \\
\hline Non-progressive motility & $66.7(2 / 3)$ & $50.0(2 / 4)$ & $80.0(4 / 5)$ & n.s \\
\hline Motility recovery after pentoxifylline treatment & $3.0 \pm 10.0(1 / 23)$ & $3.0 \pm 10.0(1 / 23)$ & $0(0 / 26)$ & n.s \\
\hline
\end{tabular}

Specimens donated from 11 patients were used in this experiment

Conventional semen parameters subjected to this analysis: total concentration $47.3 \pm 33.8 \times 10^{6}$ cells $/ \mathrm{mL}$; motile rate $53.3 \pm 18.4 \%$; abnormal morphology rate; $36.4 \pm 11.7 \%$. HA-ZP: hollow hyaluronan-phenolic hydroxyl (HA-Ph) microcapsule mimicking ZP. ZP: empty zona pellucida. Average diameter (D) and thickness of HA gel (M) in small HA-ZP was $270.0 \pm 12.4 \mu \mathrm{m}$ and $29.3 \pm 8.1 \mu \mathrm{m}$, and large HA-ZP was $327.0 \pm 13.1 \mu \mathrm{m}$ and 22.8 $\pm 8.6 \mu \mathrm{m}$, respectively. Sperm with progressive or non-progressive motility were decided as motile sperm. Total motility: total number of sperm with progressive and non-progressive motility. Motility after pentoxifylline treatment: immotile sperm after thawing of vessels was treated by pentoxifylline, and motility recovery was assessed

$n . s$. non-significant, $N=11$, mean $\pm \mathrm{SD}$

ICSI procedure. Thus, the influence of hyaluronidase treatment on sperm was investigated. We showed that treatment with hyaluronidase did not impair sperm motility or viability. This result indicated that hyaluronidase could be used for the recovery of sperm, and HA was decided as the material for the microcapsules. Hyaluronidase is generally used for denuding cumulus cells from oocytes during assisted reproductive techniques (ART). Furthermore, there have been no reports to indicate that treatment with hyaluronidase impairs embryo growth. This experience of usage during ART ensures safety. In the selection of sperm recovery method, one possible method for sperm recovery is aspiration from the capsule, but it is difficult to finely control the aspiration of sperm in a several hundred- $\mu \mathrm{m}$ capsule. In general, the procedure for sperm selection or immobilization for ICSI, the touch of a sperm head using the injection pipet, was forbidden because there was a possibility that the nuclear structure is destroyed by physical pressure. Thus, recovery by decomposition of the microcapsule was considered as best clinical practice.

The recovery rate of sperm after freeze-thawing and decomposition of the HA-ZP was almost the same as the case of freezing in a hollow $\mathrm{ZP}$, and the values were acceptable for clinical practice. Cohen et al. [12] and Ye et al. [30] indicated that the sperm recovery rates after freezing and thawing of empty ZP were 70.0 and $78.2 \%$, respectively. The recovery rate in our study was higher than their values because their method of cytoplasm extraction to prepare empty ZP was performed using a pipet larger $(15 \mu \mathrm{m})$ than a conventional injection pipet. These results suggested that the size of the hole to evacuate the ZP affects the recovery rate. In the case of the HA capsule, the evacuation procedure using a larger pipet is not needed. In addition, the injection of sperm into the HA capsule is performed using a conventional ICSI pipet. Thus, the injected sperm were stably held in the capsule.
Fig. 5 Searching time for a sperm in each sperm concentration. The vertical axis shows the time taken in searching for a sperm under each condition. Hollow hyaluronan microcapsule mimicking zona pellucida (HA-ZP) on the horizontal axis indicates the case of sperm recovered by injection into HA microcapsules and after decomposition. Different letters indicate statistically significant differences $(P<0.05)$ by the Tukey-Kramer method following $\operatorname{ANOVA}(N=3 ;$ mean $\pm \mathrm{SD})$

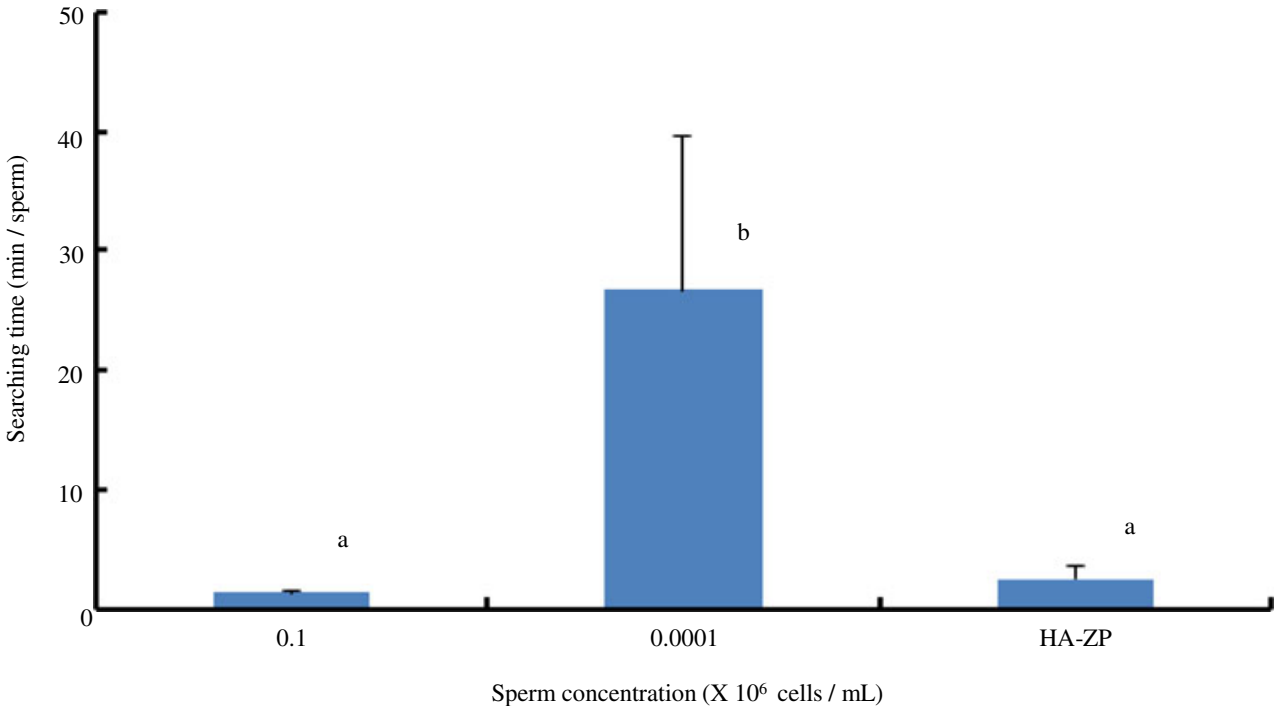

Sperm concentration $\left(\mathrm{X} 10^{6}\right.$ cells $\left./ \mathrm{mL}\right)$ 
Injection of sperm into the HA-ZP without freezing did not impair sperm motility. In the HA-ZP preparation, HA was gelated by an oxidative reaction via HRP. Subsequently, trypsin, a serine protease, was used to degrade the gelatin core of the HA microcapsule. Our results indicated that the use of these enzymes in microcapsule fabrication did not affect sperm motility. Furthermore, as animal level experiment, we confirmed whether the injection of sperm to HA-ZP and decomposition inhibit sperm fertilization (data not shown). The frozen-thawed porcine sperm injected to HA-ZP, and after capsule decomposition sperm was injected to porcine oocyte. As a result, we could observe normal fertilization in sperm in HA-ZP. These results suggested that sperm treatment by HAZP does not inhibit sperm motility and fertilization. Moreover, motility after freezing-thawing and decomposition of the HAZP was not different compared with empty ZP controls. This result indicated that freezing sperm in the HA-ZP also does not impair sperm motility. In a previous study, sodium alginate was used to encapsulate sperm. Herrler et al. [11] showed that liquid and polymerization of alginic acid in sperm encapsulation decreased sperm motility. They suggested that sperm motility was inhibited because of damage of membranes caused by $\mathrm{pH}$ changes during polymerization. In our method, because the gelation of capsules was performed before sperm injection, the step of capsule gelation did not affect sperm motility. In a preliminary experiment, we confirmed that sperm injected into HA microcapsules with gelatin cores retained their motility after decomposition, indicating that gelatin did not impair sperm motility. However, sperm motility after injection into trypsin-untreated HA microcapsules and after freezing and thawing was relatively low compared with sperm frozen in an empty ZP (HA: $7.7 \pm 14.6 \%$ vs. ZP: $22.9 \pm 29.0 \%$, n.s.). Because the aggregation of gelatin was observed in trypsinuntreated HA capsules after freezing and thawing, it was considered that aggregated gelatin by freezing caused damage to sperm plasma membranes. Thus, the removal of gelatin from the core of HA microcapsules was effective in maintaining greater sperm motility after freeze-thawing.

The motile rate of sperm after freezing-thawing in HA-ZP was about $14 \%$. The recovery rate of motile sperm is relatively low, compared with previous report of small number of sperm freezing [15]. One possibility of such value is experimental regulation of our ART center. We can use the surplus sperm specimens for IVF after finishing insemination on the day. These specimens were incubated for 3 to $5 \mathrm{~h}$ in $5 \% \mathrm{O} 2$ in $37^{\circ} \mathrm{C}$ after semen preparation. It is known that incubation of sperm for long term in vitro leads to lipid peroxidation $[5,6]$, which involves to cryosurvival [31]. Thus, in our experimental condition, sperm would decline to freezing stress. Regarding of validness of freezing method, we selected the freezing by non-permeating cryoprotectant (sucrose) [23]. One advantage of using sugar as cryoprotectant is that sperm freezing can be performed under low osmotic pressure.
According to our preliminary data, motile sperm can maintain $80 \%$ of motility under $360 \mathrm{mOsm} / \mathrm{kg}$ osmotic pressure of culture medium with $0.1 \mathrm{M}$ sucrose, but more than $50 \%$ of motile sperm lose the motility under over $490 \mathrm{mOsm} / \mathrm{kg}$. Due to osmotic pressure of general sperm freezing medium is about $1500 \mathrm{mOsm} / \mathrm{kg}$, it was considered that more motile sperm can be obtained by sugar based freezing medium. Other advantage is that, in the rapid freezing condition, freezing medium with low concentration of cryoprotectant is suitable rather than that with conventional concentration (glycerol based freezing medium) [32]. Our preliminary data showed that sperm motility after freezing in empty ZP on Cryotop by $0.1 \mathrm{M}$ sucrose group tend to be higher than $7 \%$ glycerin group ( 23.1 vs. $7.5 \%$, n.s., $N=4$ ). Therefore, we considered that freezing procedure by sucrose based freezing medium is proper method, compared with conventional freezing solution.

There was difference of diameter in prepared HA capsules. The size difference was arisen in same and different lot of prepared capsule sample. To investigate whether the capsule size affect to the recovery rate of motile sperm, we picked up smaller and larger HA capsules from same lot of prepared sample. We had hypothesis that if the capsule size difference affect to thermal conductivity, the capacity of sperm freezing will be different. However, actual thermal conductivity cannot be measured in the vessel of micrometer order. As a result, there was no difference in the total motility and grade motility between small and large HA capsule groups. Herrler et al. [11] showed that the sperm motility after embedding freezing in different size of alginic acid gel in same volume of freezing medium was not different. Isachenko et al. [19] indicated that motility after freezing in different volume of sperm suspension was significantly different. These reports suggested that whole volume of freezing medium is critical for motile sperm recovery after freezing. In our method, the diameter difference of HA capsule used for sperm freezing is several tens of micrometers. Such size difference did not affect to sperm motility after freezing-thawing. We also showed that the motility recovery of immotile sperm after pentoxifylline treatment was low in all vessels, and there was no difference among vessels. This result indicated immotile sperm in our freezing method has no potential to be injected to oocyte. Some reports showed that treatment of pentoxifylline after freezing-thawing of human sperm increases motile rate [24, 33]. Motility recovery indicates that immotile sperm retained motility potential. Such motility recovery rate after freezing involves protective ability of freezing method. Thus, we need to modify the freezing method for improvement of motility recovery of immotile sperm after freezing.

Searching time for a sperm from an HA-ZP was equivalent to that of a general concentration of sperm used in ICSI. This result indicated that, even in the case of severe oligozoospermia or TESE ICSI, once sperm are held in the HA-ZP, they can be recovered more quickly than using conventional methods of 
sperm freezing. Clinical data in our laboratory showed that sperm searching time in TESE ICSI, the ability to find a few sperm in a dish before cryopreservation, took $20 \mathrm{~min}$. This value was longer than the case which can find at least a sperm in a field in $\times 400$ magnification ( $5 \mathrm{~min}$ ). Thus, freezing using HA microcapsules is most effective in such TESE case with a few sperm for reducing sperm searching time of ICSI.

In the present study, a small number of sperm could be held in HA hollow microcapsules using conventional ICSI procedures. After thawing and decomposition of the capsule using hyaluronidase, a good recovery rates was obtained. In addition, the searching time for sperm held in HA capsules was equivalent to that for normal ICSI concentration samples. However, the potential for fertilization and embryo growth after using sperm frozen with HA hollow microcapsules has not been investigated. Animal level studies are needed to test HA microcapsules before they are introduced into clinical practice.

\section{Acknowledgments None}

\section{Compliance with ethical standards}

Conflict of interest The authors declare no conflict of interests

\section{References}

1. Palermo G, Joris H, Devroey P, Van Steirteghem AC. Pregnancies after intracytoplasmic injection of single spermatozoon into oocyte. Lancet. 1992;340:17-8.

2. Silber SJ, Nagy ZP, Liu J, Godoy H, Devroey P, Van Steirteghem AC. Conventional in-vitro fertilization versus intracytoplasmic sperm injection for patients requiring microsurgical sperm aspiration. Hum Reprod. 1994;9:1705-9.

3. Lyu BN, Ismailov SB, Ismailov B, Lyu MB. Mitochondrial concept of leukemogenesis: key role of oxygen-peroxide effects. TheorBiol Med Model. 2008;5:23. doi:10.1186/1742-4682-5-23.

4. Ford WC. Regulation of sperm function by reactive oxygen species. Hum Reprod Update. 2004;10:387-99.

5. Alvarez JG, Storey BT. Lipid peroxidation and the reactions of superoxide and hydrogen peroxide in mouse spermatozoa. Biol Reprod. 1984;30:833-41.

6. Alvarez JG, Touchstone JC, Blasco L, Storey BT. Spontaneous lipid peroxidation and production of hydrogen peroxide and superoxide in human spermatozoa. Superoxide disumutase as major enzyme protectant against oxygen toxicity. J Androl. 1987;8:338-48.

7. Fujitani Y, Kasai K, Ohtani S, Nishimura K, Yamada M, Utsumi K. Effect of oxygen concentration and free radicals on in vitro development of in vitro-produced bovine embryos. J Anim Sci. 1997;75: 483-9.

8. Matsuura R, Takeuchi T, Yoshida A. Preparation and incubation conditions affect the DNA integrity of ejaculated human spermatozoa. Asian J Androl. 2010;12:753-9.

9. Desai NN, Blackmon H, Goldfarb J. Single sperm cryopreservation on cryoloops: an alternative to hamster zona for freezing individual spermatozoa. Reprod Biomed Online. 2004;9:47-53.
10. Endo Y, Fujii Y, Kurotsuchi S, Motoyama H, Funahashi H. Successful delivery derived from vitrified-warmed spermatozoa from a patient with nonobstructive azoospermia. Fertil Steril. 2012;98:1423-7.

11. Herrler A, Eisner S, Bach V, Weissenborn U, Beier HM. Cryopreservation of spermatozoa in alginic acid capsules. Fertil Steril. 2006;85:208-13.

12. Cohen J, Garrisi GJ, Congedo-Ferrara TA, Kieck KA, Schimmel TW, Scott RT. Cryopreservation of single human spermatozoa. Hum Reprod. 1997;12:994-1101.

13. Walmsley R, Cohen J, Ferrara-Congedo T, Reing A, Garrisi J. The first births and ongoing pregnancies associated with sperm cryopreservation within evacuated egg zonae. Hum Reprod. 1998;Suppl 4:61-70.

14. Hsieh Y, Tsai H, Chang C, Lo H. Cryopreservation of human spermatozoa within human or mouse empty zona pelllucidae. Fertil Steril. 2000;73:694-8.

15. AbdelHafez F, Bedaiwy M, El-Nashar SA, Sabanegh E, Desai N. Techniques for cryopreservation of individual or small numbers of human spermatozoa: a systematic review. Hum Reprod Update. 2009;15:153-64.

16. Kurisawa M, Chung JE, Yang YY, Gao SJ, Uyama H. Injectable biodegradable hydrogels composed of hyaluronic acid-tyramine conjugates for drug delivery and tissue engineering. Chem Commun (Camb). 2005;14:4312-4.

17. Sakai S, Hashimoto I, Ogushi Y, Kawakami K. Peroxidasecatalyzed cell encapsulation in subsieve-size capsules of alginate with phenol moieties in water-immiscible fluid dissolving $\mathrm{H}_{2} \mathrm{O}_{2}$. Biomacromolecules. 2007;8:2622-6.

18. Aitken RJ, Baker HWG, Barratt CLR, Behre HM, Comhaire F, Cooper TG, De Jonge C, Eliasson R, Farley TMM, Griffin PD, et al. WHO laboratory manual for the examination of human semen and sperm-cervical mucus interaction. 4th edn, Press syndicate of the University of Cambrige, Cambridge UK; 2000. pp. 34-35.

19. Isachenko E, Isachenko V, Weiss JM, Kreienberg R, Katkov II, Schulz M, et al. Acrosomal status and mitochondrial activity of human spermatozoa vitrified with sucrose. Reproduction. 2008;136:167-73.

20. Zhu J, Jin RT, Wu LM, Johansson L, Guo TH, Liu YS, et al. Cryoprotectant-free ultra-rapid freezing of human spermatozoa in cryogenic vials. Andrologia. 2014;46:642-9.

21. Isachenko V, Isachenko E, Katkov II, Montaq M, Dessole S, Nawroth F, et al. Cryoprotectant-free cryopreservation of human spermatozoa by vitrification and freezing in vapor: effect on motility, DNA integrity, and fertilization ability. Biol Reprod. 2004;71: 1167-73.

22. Isachenko V, Isachenko E, Petrunkina AM, Sanchez R. Human spermatozoa vitrified in the absence of permeable cryoprotectants: birth of two healthy babies. Reprod Fertil Dev. 2012;24:323-6.

23. Hossain AM, Osuamkpe CO. Sole use of sucrose in human sperm cryopreservation. Arch Androl. 2007;53:99-103.

24. Tasdemir I, Tasdemir M, Tavukcuogiu S. Effect of pentoxifilline on immotile testicular spermatozoa. J Assist Reprod Genet. 1998;15:90-2.

25. Ganan-Calvo AM, Gordillo JM. Perfectly monodisperse microbubbling by capillary flow focusing. Phys Rev Lett. 2001;87:274501.

26. Sakai S, Kawabata K, Ono T, Iijima H, Kawakami K. Preparation of mammalian cell-enclosing subsieve-sized capsules $(<100 \mu \mathrm{m})$ in a coflowing stream. Biotechnol Bioeng. 2004;86:168-73.

27. Ribeiro AJ, Neufeld RJ, Arnaud P, Chaumeil JC. Microencapsulation of lipophilic drugs in chitosan-coated alginate microspheres. Int J Pharm. 1999;187:115-23. 
28. Lim ST, Martin GP, Berry DJ, Brown MB. Preparation and evaluation of the in vitro drug release properties and mucoadheasion of novel microspheres of hyaluronic acid and chitosan. J Control Release. 2000;66:281-91.

29. Sakai S, Ito S, Ogushi Y, Hashimoto I, Hosoda N, Sawae Y, et al. Enzymatically fabricated and degradable microcapsules for production of multicellular spheroids with well-defined diameters of less than $150 \mu \mathrm{m}$. Biomaterials. 2009;30:5937-42.

30. Ye Y, Xu C, Qian Y, Jin F, Huang H. Evaluation of human sperm function after being cryopreserved within the zona pellucida. Fertil Steril. 2009;92:1002-8.
31. Deqllnnocenti S, Filimberti E, Maqini A, Krausz C, Lombardi G, Fino $\mathrm{MG}$, et al. Semen cryopreservation for men banking for oligospermia, cancers, and other pathologies: prediction of post-thaw outcome using basal semen quality. Fertil Steril. 2013;100:1555-63.

32. Isachenko E, Isachenko V, Katkov II, Rahimi G, Schondorf T, Mallmann P, et al. DNA integrity and motility of human spermatozoa after standard slow freezing versus cryoprotectant-free vitrification. Hum Reprod. 2004;19:932-9.

33. Stanic P, Sonicki Z, Suchanek E. Effect of pentoxifylline on motility and membrane integrity of cryopreserved human spermatozoa. Int J Androl. 2002;25:186-90. 